\title{
Generating an image that affords slant perception from stereo, without pictorial cues
}

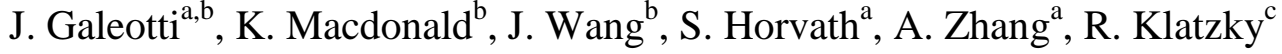 \\ ${ }^{a}$ Robotics Institute, Carnegie Mellon University, Pittsburgh, PA, 15213, USA, \\ jgaleotti@cmu.edu, shorvath@cmu.edu, ajzhang@cs.cmu.edu \\ ${ }^{\mathrm{b}}$ Dept. of Biomedical Engineering, University of Pittsburgh, Pittsburgh, PA, \\ 15261,USA,kam233@pitt.edu,jiw86@pitt.edu \\ ${ }^{c}$ Dept. of Psychology and Human-Computer Interaction Institute, Carnegie Mellon \\ University, Pittsburgh, PA, 15213, USA, klatzky@cmu.edu
}

\author{
Corresponding Author \\ Dr. John Galeotti \\ Carnegie Mellon University \\ Robotics Institute, NSH A525 \\ Pittsburgh, PA 15213 \\ $+14125599351$ \\ jgaleotti@cmu.edu
}

\begin{abstract}
This paper describes an algorithm for generating a planar image that when tilted provides stereo cues to slant, without contamination from pictorial gradients. As the stimuli derived from this image are ultimately intended for use in studies of slant perception under magnification, a further requirement is that the generated image be suitable for high-definition printing or display on a monitor. A first stage generates an image consisting of overlapping edges with sufficient density that when zoomed, edges that nearly span the original scale are replaced with newly emergent content that leaves the visible edge statistics unchanged. A second stage reduces intensity clumping while preserving edges by enforcing a broad dynamic range across the image. Spectral analyses demonstrate that the
\end{abstract}


low-frequency content of the resulting image, which would correspond to the pictorial cue of texture gradient changes under slant, (a) has a power fall-off deviating from $1 / \mathrm{f}$ noise (to which the visual system is particularly sensitive), and (b) does not offer systematic cues under changes in scale or slant. Two behavioral experiments tested whether the algorithm generates stimuli that offer cues to slant under stereo viewing only, and not when disparities are eliminated. With a particular adjustment of dynamic range (and nearly so with the other version that was tested), participants viewing without stereo cues were essentially unable to discriminate slanted from flat (frontal) stimuli, and when slant was reported, they failed to discriminate its direction. In contrast, non-stereo viewing of a control stimulus with pictorial cues, as well as stereoscopic observation, consistently allowed participants to perceive slant correctly. Experiment 2 further showed that these results generalized across a population of different stimuli from the same generation process and demonstrated that the process did not substitute biased slant cues.

Keywords texture gradients; depth perception; pictorial cues; stereopsis; fractals; slant perception

\section{Introduction}

When people view a surface that deviates from a perpendicular along the line of sight, they have available three classes of cues to judge its slant ${ }^{1}$ : the so-called pictorial cues, which would be visible with monocular viewing; stereoscopic disparities, which are available only with two eyes; and oculomotor cues of convergence and accommodation. (Depending on viewing conditions, movementbased cues may also be available.) Considerable research on this topic has concentrated on the pictorial cues; more specifically, on how slant is signaled by gradient changes in spatial elements across a pictorial display. Gibson [1] famously used the term texture gradient to capture the progressive change in image statistics with distance from the viewer. Purdy, Gibson's student, attempted in his thesis work [2] to characterize gradient cues more systematically

\footnotetext{
${ }^{1}$ The term slant is used here for rotations of an image relative to the frontoparallel plane, with the rotation axis centered on the image and oriented either horizontally (pitch rotation) or vertically (yaw rotation).
} 
and found that several candidate properties of regular texture elements, such as principal axes and their ratios, were powerful cues to slant. In more recent research, Todd and associates $[\mathbf{3 , 4}]$ have argued that local analysis is insufficient to compute slant from objectively 2D textures; region-based metrics are needed.

Research on pictorial gradients seeks to understand how surface orientation is computed in the absence of stereo disparities, which would directly convey that points on a slanted surface vary in depth from the viewer. In circumstances where judgments are made from a 2D display, this constitutes a cue-conflict situation, because pictorial cues in a 2D picture will necessarily be in conflict with stereoscopic and oculomotor cues, both of which would indicate the picture lies in the frontal plane. Even if one eye is closed, there is still a conflict between accommodation and pictorial cues in a $2 \mathrm{D}$ image.

Relatively little research has addressed the reverse problem, namely, how stereo disparities can cue slant in the absence of pictorial cues. The goal of the present research was to develop an algorithm for generating an image that would approximate statistical invariance over a large range of scales, and hence not give rise to gradient cues when slanted, while still affording stereo perception of local depth - and hence slant. In contrast to classical scale space $[\mathbf{5 , 6}]$, in which statistics change across scale (typically monotonically due to Gaussian blurring), we are trying to maintain invariant statistics across scale.

Zabulis and Backus [7] offered one approach to this problem, "Starry Night" textures, composed of points varying in flux. Pictorial cues of size and perspective were avoided by adjusting each point in the texture to a circular dot of constant radius. While this dynamic approach is possible when the displays are computer-generated, one goal of our research is to eventually produce stimuli that could be printed and presented under a microscope at different slants, in order to investigate how well slant can be perceived under conditions of magnified viewing from stereo cues alone.

This goal led us to develop an algorithm for generating images with three critical properties: First, as the detection of stereo correspondence is facilitated by the 
uniqueness of shape features and the continuity of edges (e.g, Marr \& Poggio [8]), there should be dense edge content. Second, in order to isolate the contribution of stereo cues, perspective changes under slant should be avoided (although foreshortening alone may not be sufficient to induce slant perception [9]). Third, given the known importance of gradient changes for pictorial slant perception, perceivable edge density should remain constant as the image is rotated relative to the viewer's line of sight. At a local level, rotation can be treated as a change in scale. Thus a critical feature of the generation algorithm, especially given our interest in slant under magnified viewing, is that the statistics of the resulting images are not indicative of viewing scale. In particular, as will be discussed later, the algorithm is constructed with the goal that the edge statistics (i.e., statistics of high-frequency content) are scale-invariant, while texture gradient information is suppressed by perturbation of low frequency content.

The goal of scale invariance suggests the use of a fractal image, which is scale invariant by definition. However, fractals are constrained only to have equivalent non-regularity across scales, and as is exhibited by well-known fractal sets $[\mathbf{1 0 , 1 1}]$, sub-regions can have relatively sparse, object-like shapes, the density of which changes with rescaling. The approach taken here was to determine a means of generating images with the desired properties de novo and then to test the extent to which they cued slant. Under the goal of scale invariance, the generated content consisted of overlapping edges with sufficient density that when zoomed in, edges that nearly spanned the original scale disappeared, but were replaced with newly emergent edge content that left the statistics unchanged. (These newly emergent edges are always in the generated image, but given the resolution limits of either a human eye or a computer monitor, they are too small to see when zoomed out.)

\section{Image Generation}

\subsection{Generation Algorithm}

In general, if a source image is viewed within a rectangular aperture, zooming in by a factor of $\mathrm{k}$ will reduce the viewed source area to $1 / \mathrm{k}^{2}$ of its previous value, while the source data that are currently in view will now be assigned $\mathrm{k}^{2}$ its 
previous number of pixels. Maintaining invariance under zoom thus requires $\mathrm{k}^{2}$ times as many emergent edges in the entire image as there are vanishing edges.

To achieve this goal, the algorithm additively superimposes a large number of randomly shaped and oriented, overlapping triangles, where the scales of the triangles are distributed so as to maintain the inverse-square law. Shapes are used as the route to edges, because a directly generated edge is a 2D shape (albeit very small on one of the dimensions) that will change in size as it is rescaled.

Triangles, in particular, were chosen as edge generators because the literature on slant from texture indicates certain shapes that are precluded a priori, particularly circles and rectangles, which will create undesirable perspective cues in the image, as well as shapes with constant parameters such as ellipses with a common axis ratio, and even families of blobs with underlying statistical regularities.

The process of generating a single triangle is illustrated by Figure 1: A circle (not rendered) of radius $r$ was placed at a randomly selected point, three points were randomly selected around the circle, and tangent lines were drawn to these points, with the intersections constituting the three vertices of the triangle. Additional constraints were placed on the configuration so that the tangents intersected within a reasonable distance, avoiding near-parallel edges: Relative to the first point of tangency $\left(\mathrm{p}_{1}\right), \mathrm{p}_{2}$ was restricted to angular distances in the range $<\left(15^{\circ}\right.$ $\left.165^{\circ}\right),\left(195^{\circ}-345^{\circ}\right)>$, and $\mathrm{p}_{3}$ must fall on the larger arc formed between $\mathrm{p}_{1}$ and $\mathrm{p}_{2}$ such that (1) the resulting triangle will contain the inscribed circle and (2) $\mathrm{p}_{3}$ will not fall within $20^{\circ}$ of $\mathrm{p}_{1}$ or $\mathrm{p}_{2}$.

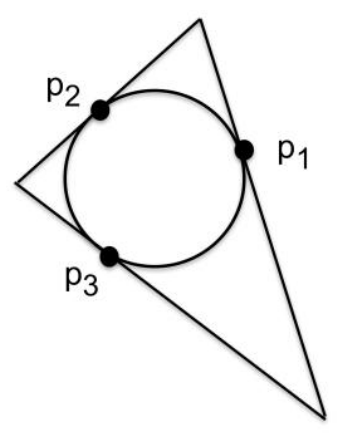

Figure 1. Triangle generated from three tangent lines. The circle is not rendered; see text for description. 
The scale of a triangle is defined as the radius of the largest circle that can be inscribed within it. To produce a distribution of scales, radii of circles used to generate triangles were drawn from a distribution of sizes according to

$$
r=\frac{a}{\left(\frac{a}{r_{\min }}-\gamma\right)}
$$

where $a$ is defined as:

$$
a=\frac{1}{\frac{1}{r_{\min }}-\frac{1}{r_{\max }}}
$$

In (1) $r$ represents the radius of the circle to be plotted, and $r_{\min }$ and $r_{\max }$ are the limits of the radii allowed. The variable $\gamma$ is drawn from a uniform distribution between 0 and 1. Equation (1) achieves the goal of biasing the triangle distribution toward small scales.

To localize the triangles within the image, the centers of generating circles were placed randomly over its area. An extension on the boundaries was included in the computation as needed to generate triangles centered beyond them, so as to create uniform content, even at the extremities. The brightness values were then determined by additively superimposing the triangles, according to the rule that each pixel's final intensity equals the number of triangles covering it. Fifteen million triangles were sufficient to generate a "source image" at a resolution of 45,600 pixels wide by 31,200 pixels high with a broad range of intensities. The source size was selected to allow printing at a large $(12+$ inch $)$ size. The large source image also allows us to cut smaller patches that can be used to create experimental stimuli, either by printing or presenting on a monitor. In order for the displayed stimulus to exhibit the properties intended by the generation algorithm, its pixilation must be imperceptible to the observer under all viewing conditions across our range of slant. For our experiments, the monitor pixel size and viewing distance must be implemented so as to ensure that this goal is met. Another important constraint that applies to printed and computer-generated stimuli is that the pixel size of the source image must be as small as or smaller than the display pixel size. Higher-resolution source images are also advantageous to reduce quantization error when down-sampling. For the present studies, the goal of an ample margin of safety for pixel resolution and 
quantization, along with the requirement that the dimensions of the displayed stimulus must be a power of 2 for rendering purposes, led us to a resolution of 1 screen pixel to 3.1 source pixels for a stimulus presented en face. A tilted stimulus has a slightly lower/higher pixel ratio for the portions of the stimulus that are tilted toward/away from the viewer, respectively.

As will be shown in the analyses below, the result of the algorithm up to this point has a frequency spectrum in which spectral power decreases with frequency, as is to be expected from fractal content. The high power in the low-frequency components of the spectrum reflects random clustering of overlapping triangles in space (Poisson clumping), which is observable in initially generated images as visible patches of predominantly bright or dark regions. The contrast between these clumps was reduced under zooming, providing a potential gradient cue.

To eliminate this cue, a second step in the algorithm enforced dynamic range across all portions of the image. Specifically, the pixel values resulting from the addition of the triangles were remapped by a quantized repeating sinusoid with a phase shift of $\pi$ and a frequency of 4 (i.e., the remapped pixel values wrap around between white and black 4 times across the range of original values). To accommodate to the dynamic range of a 3D monitor and avoid washout or blackness, the maximum and minimum values were curtailed, rather than reaching black (digital 0) and white (digital 255). The curtailed range was an experimental parameter here: In the generated version called wrap 1, the brightness values varied from 8 to 248, whereas in generated version wrap 2, they varied from 16 to 240 , in both cases in increments of 8 . The equations for the remapping step are:

$$
\begin{gathered}
\mathrm{I}_{\mathrm{W}}=8 * \operatorname{round}\left(16+\beta \sin \left(2 \pi f \mathrm{I}_{\mathrm{s}}-\pi\right)\right) \\
\beta=16-\frac{\mathrm{p}_{\min }}{8} \\
\mathrm{I}_{\mathrm{W}} \text { : wrapped image } \\
\mathrm{p}_{\mathrm{sin}}: \text { input image scaled to } 0-1 \text { range } \\
\beta: \text { constant to rescale to desired region } \\
\text { f: frequency in intensity space }
\end{gathered}
$$



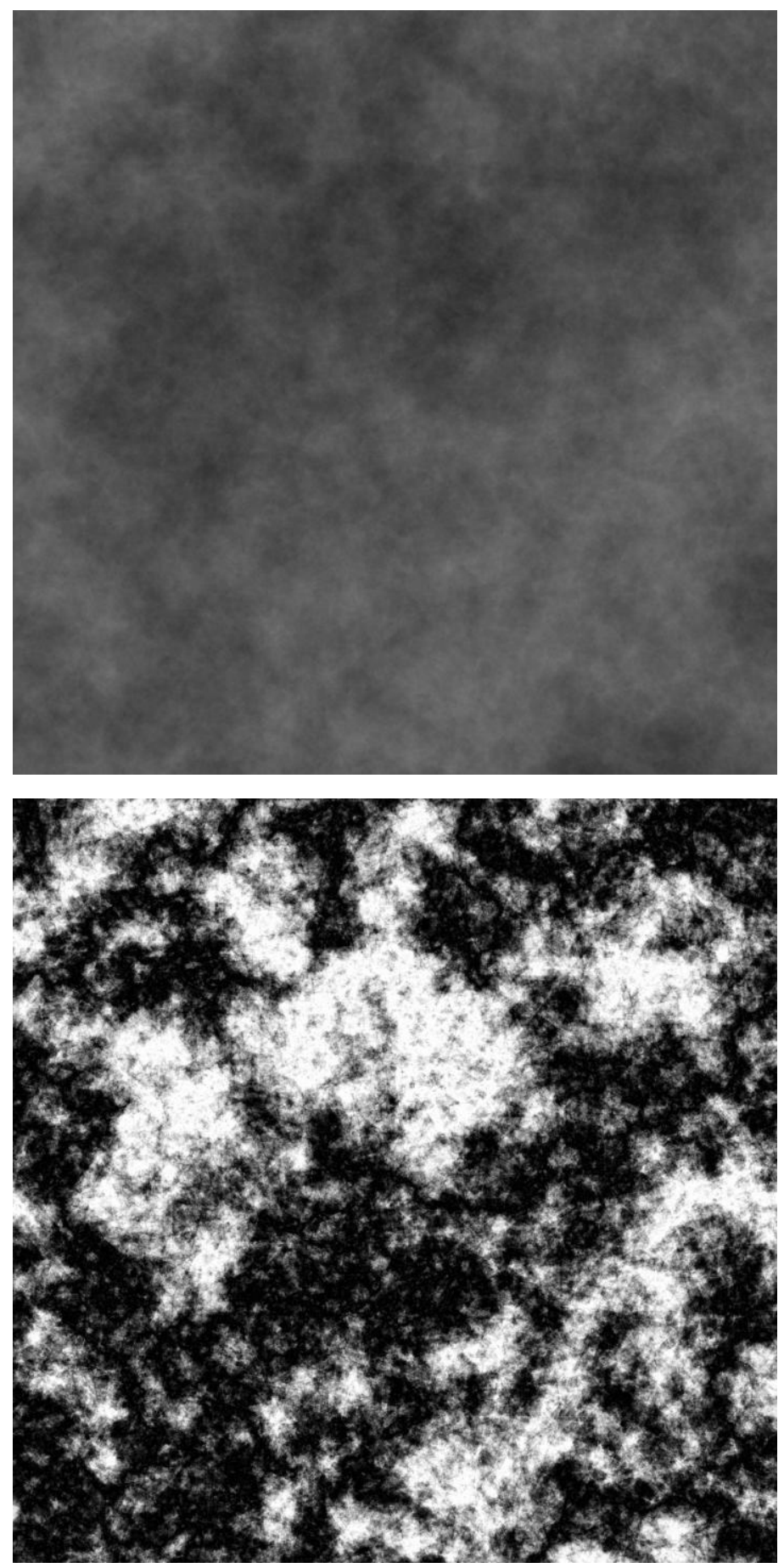

Figure 2. Algorithm-generated patches before (top) and after (bottom) sinusoidal remapping. Before remapping, random Poisson clumping caused the right half of the top figure to contain substantially more dark pixels than the left half. After remapping, there are bright and dark clumps of varying sizes spread throughout the area.

The parameter controlling the wrap value, $\mathrm{p}_{\min }$, is set to 8 for wrap 1 and 16 for wrap 2. The standard decoding gamma (2.2) for Windows sRGB was used for both wraps. While the gamma values of monitors vary, most modern monitors can be calibrated to approximate an ideal gamma curve. Incorrect gamma levels 
can lead to loss of information in the brightest and darkest regions of the image. Sinusoidal wrapping and clamping the extremes of the intensity serve to move the behavior of the image away from the edge cases of gamma correction and make it more robust to limitations of a non-ideal monitor. Printing the wrapped image would require a professional-grade printer (Canon PRO-1 or similar), which manages its own gamma to optimally match intensities with the source data. Figure 2 shows an example of a patch from the source image after step 1, and then in the second step after remapping (wrap 1, which ultimately proved more effective for our purposes).

\subsection{Spectrum of the generated stimuli}

The visual system is known to be sensitive to power distributions with a frequency fall-off of the form $\mathrm{f}^{-\beta}[\mathbf{1 2}, \mathbf{1 3}]$, which also characterizes the power spectra of many scenes in everyday visual experience (see Billock [12] for review) as well as fractals [13]. The present images demonstrate such a power distribution before sinusoidal remapping, consistent with the fractal property of scale invariance. The value of the spectral fall-off was measured for patches drawn randomly from a source image by computing the 2D Fourier series and then converting it to a 1D (radial) spectrum. To eliminate quantization noise, this was done by extracting a neighborhood of 1D spectra around a diagonal from the center (the DC point) and averaging the power spectra [14]. The neighborhoods comprised angles of $45^{\circ}$ to $50^{\circ}$ degrees in $.25^{\circ}$ increments. The value of $\beta$ was then extracted from a linear fit to the average spectrum in $\log / \log$ space, as shown in Figure 3 (the "Before Sinusoidal Remapping" points). When this process was applied to six patches, all such fits yielded $\beta=2.8$, with $r^{2}>.98$.

Also shown in Figure 3 is the change in the spectrum after the second stage of the generation algorithm using sinusoidal remapping. This can be seen to amplify power overall, but more importantly, in comparison to higher frequencies, there is reduced relative power at the low frequencies associated with slow gradient change in the image. 


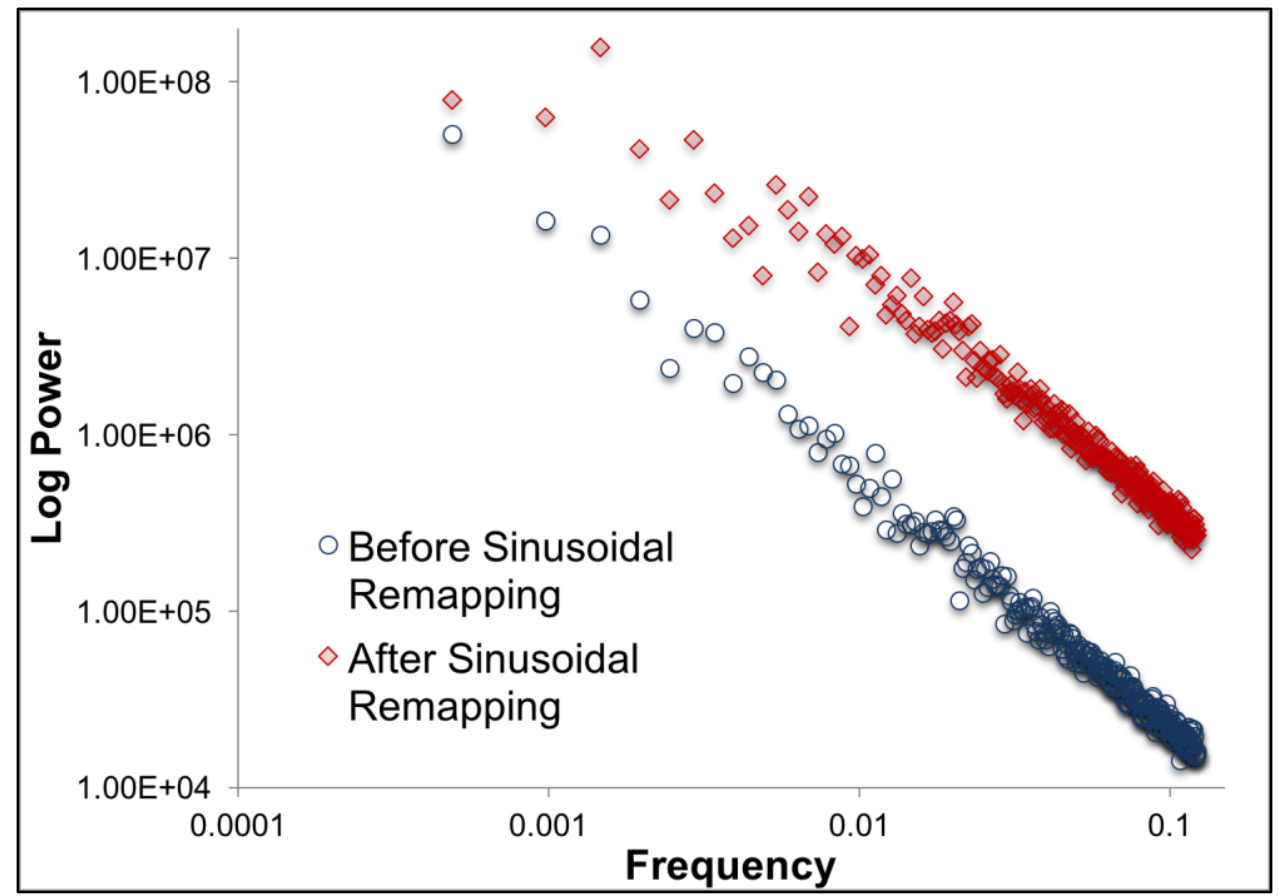

Figure 3. Power spectra of patches from a source image generated by the algorithm.

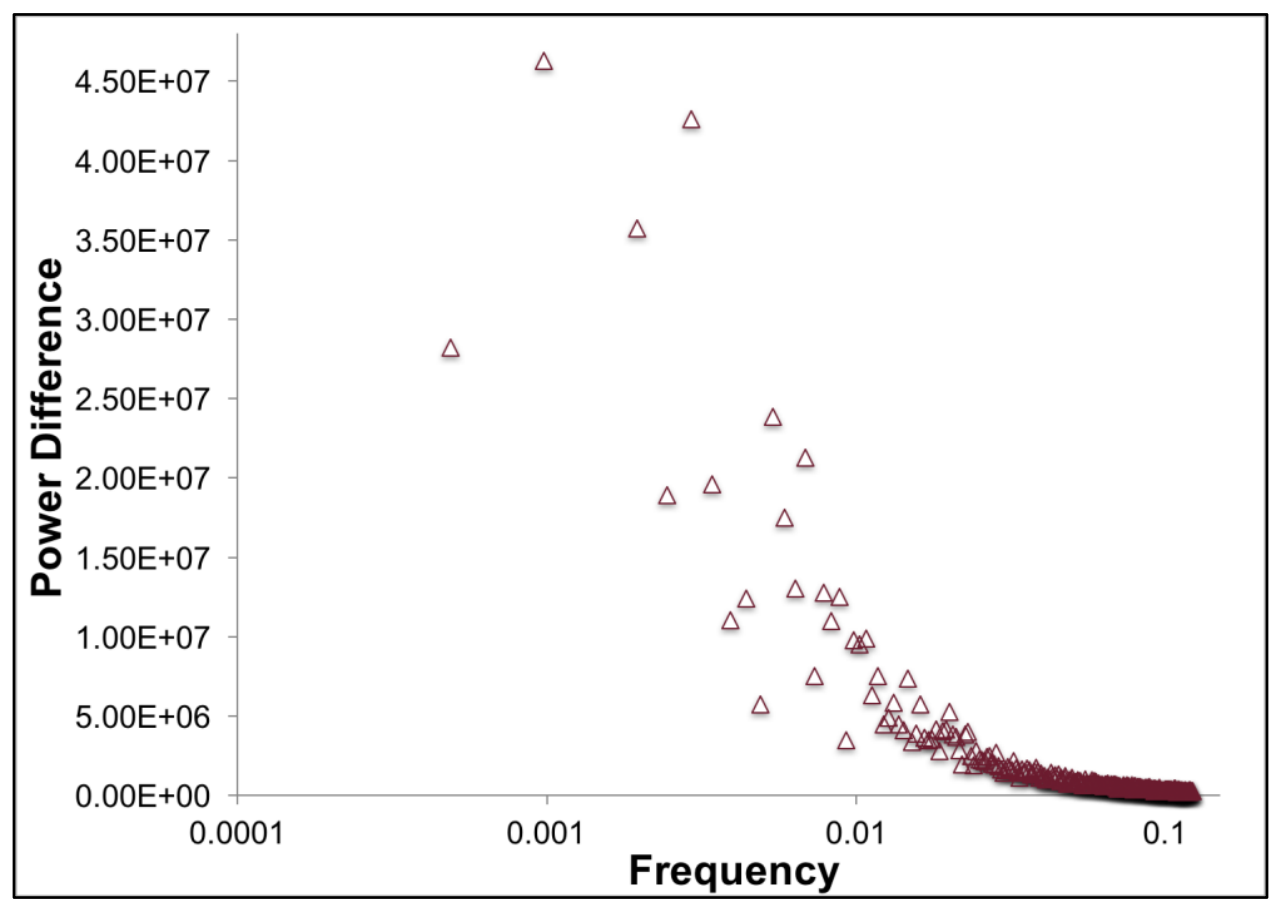

Figure 4. Difference in power of unmapped and remapped versions of a sampled patch, by spectral frequency. One outlier at the third frequency value, visible in Figure 3, is not shown.

Figure 4 shows in detail how the remapping affects the relative power spectra. Shown is the difference in spectral power between the unmapped and remapped version of the same image patch as a function of the frequency. The relative power loss due to remapping drops off sharply with frequency (essentially 
following a power function with exponent $=-1.24, \mathrm{r}^{2}=.95$ ). This trend should promote the effects desired for stimuli that convey slant through stereo alone: Preservation of the high-frequency content should retain the edge information needed for stereo matching, while disruption of the low frequencies should reduce the pictorial information from the slowly changing gradient.

\subsection{Invariance under scale}

A further analysis directly assessed the effects of scale change on the power spectra. The spectral content was analyzed at two levels of scale, corresponding to a 2x magnified zoom-in. A square image patch measuring 8,192 pixels on each side was extracted from the source image and used to create two sub-patches measuring 4,096 pixels on each side. One was created by down sampling the patch to measure 4,096 pixels on each side, and the other by cropping it to extract the center square containing 4,096 pixels on each side. (The two sub-patches, which are equated in numbers of pixels, represent two levels of scale.) This was done both for the unmapped and remapped stages of generation. Of primary interest is whether or not differences in the spectral content at the two scales are systematic, particularly at the low frequencies where gradient cues would be represented. Figure 5 shows the running variance (across 25 successive frequency levels) in the difference between power at the two scales, for both the unmapped and remapped source images. After remapping, power differences due to scale change are highly unsystematic at lower frequencies, suggesting that the local scale changes produced by slant would not be cued by graded changes in the projected stimulus, but high frequency edge content is still preserved. 


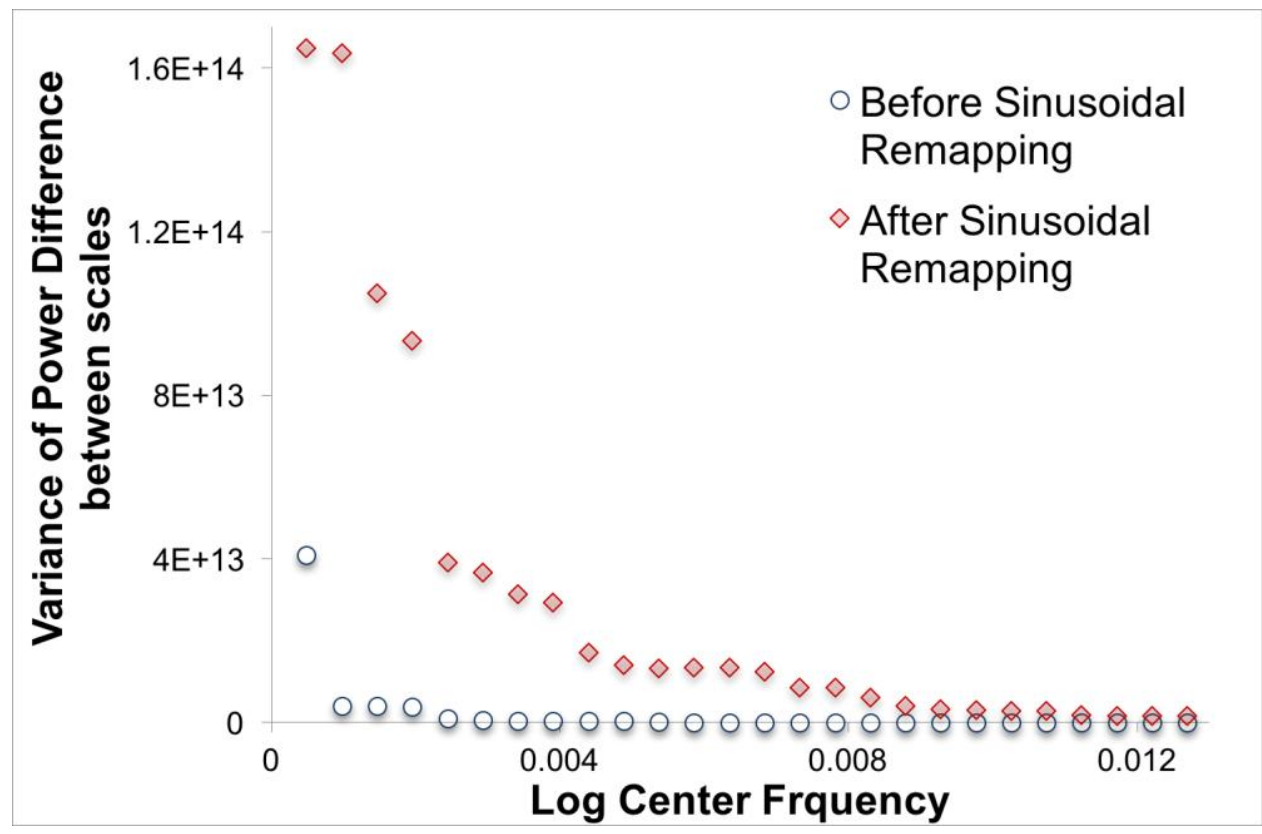

Figure 5. Running variance of power difference between scales by center frequency, for unmapped and remapped stages of generation. Scale changes in images with remapped pixel values result in highly unsystematic power differences at low frequencies, suggesting that scale changes are not inferable from image content. Since slant represents local changes in scale, it should likewise not be cued by the displayed stimulus.

\section{Experimental Overview}

The experiments assessed the efficacy of slant perception under conditions of stereo and non-stereo viewing, using algorithm-generated stimuli and, for comparison, pictures of tiles that had clear pictorial perspective cues. The task was a forced-choice judgment, in which participants viewed a stimulus display that was either flat (i.e. frontal, perpendicular to the sagittal axis) or slanted $15^{\circ}$ or $30^{\circ}$, and indicated its orientation direction by a 5 -alternative response.

For purposes of the studies, a large number of stimuli were created by cutting patches from a source image generated by our algorithm. Experiment 1 used a randomized assignment of stimuli to viewing condition, so that the measured level of slant perception would not reflect the contribution of a single stimulus. The following measures were obtained: (i) proportion of responses that were accurate as to slant direction, over all stimuli (5 possible directions) and when confining responses to stimuli reported as slanted rather than flat (4 possible slants); (ii) the ability to make a binary discrimination of stimuli into slanted vs. flat, using the d' statistic from signal detection theory (hit = slanted stimulus correctly identified, 
false alarm = flat stimulus called slanted) and (iii) a measure of information transmitted by the stimulus set in bits. The response time was also considered as a measure of the time to obtain the stereo effect.

Experiment 2 tested whether individual stimuli might offer non-stereo cues to slant, by obtaining responses from multiple participants for the same stimulus. Monte Carlo simulations were used to statistically test whether the response choices to algorithm-generated stimuli in the Mono condition were randomly distributed across the five slant alternatives. The study also provided an additional assessment of slant perception.

\section{Experiment 1}

\subsection{Participants}

The participants were 12 young adults recruited from the Carnegie Mellon University community who were paid $\$ 10$. All gave informed consent, and all experiments were conducted under IRB approval. All participants had stereo acuity of 32 seconds of arc or better, as assessed by a test (Vision Assessment Corporation).

\subsection{Stimuli}

There were two classes of stimuli, algorithm-generated and pictures of tiles. Generated stimuli (after remapping) as rendered in 3D are shown in Figure 6, top. The control stimuli were images of ceramic tiles that offered clear pictorial perspective cues, as shown rendered in 3D in Figure 6, bottom. The on-screen size of a single tile was approximately $1.1 \mathrm{~cm}$ (38.7 pixels), and the grayscale values were broadly distributed with a modal value of approximately 130 (where "black" $=0$ and "white" $=255$ ). 


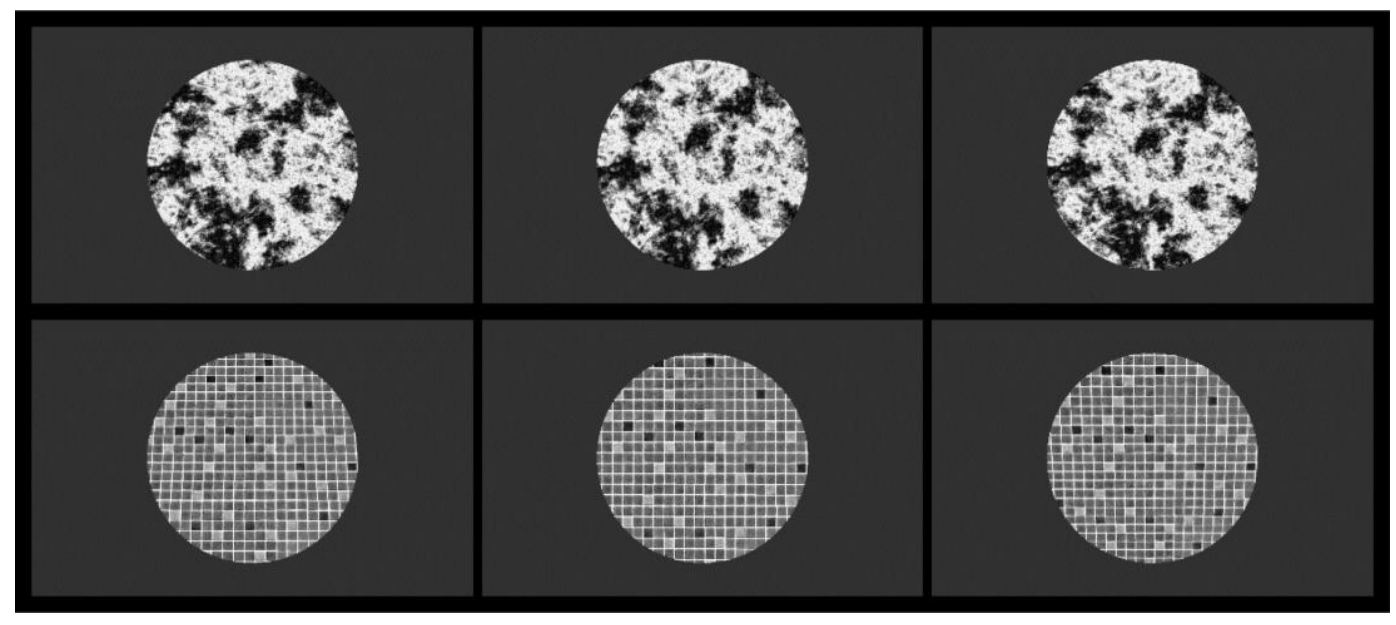

Figure 6. Upper and lower rows show generated and control stimuli, respectively. Each column shows a different slant value: from left to right, they are top slanted inward (away from the viewer) $30^{\circ}$, flat, and bottom inward $30^{\circ}$.

The generated stimulus set was constructed by extracting square patches from a large source image generated by the algorithm. The set comprised 6 sub-sets, as defined by two variables. The first was the wrapping parameter used in generation ( 2 possible parameter values), which affected the dynamic range of the source image as described previously. The second variable was the region of the patch in the generated source image. Specifically, slanted patches were rendered in $3 \mathrm{D}$ by rotating the generated source image around a line of bisection and dividing each half into thirds. For a given patch, its value on the region variable refers to whether it was drawn from the third that was nearest, in the middle, or furthest from the rotation axis. This variable was examined because any gradient cues in the source image should be strongest in the region nearest to the rotation axis.
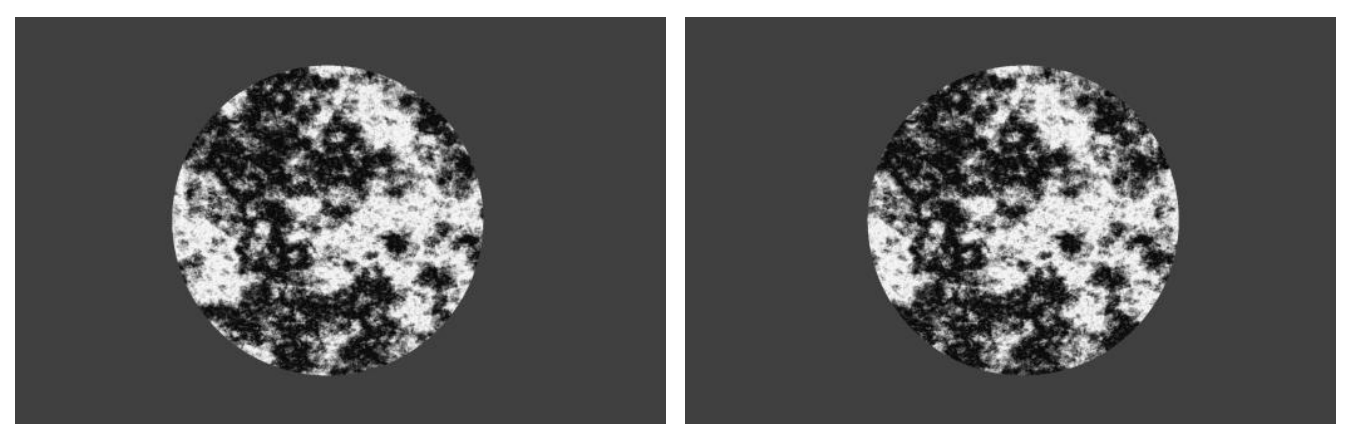

Figure 7. Separate left- and right-eye views of a generated stimulus, shown rendered in stereoscopic $3 \mathrm{D}$ with the bottom slanted inward $30^{\circ}$. 
For purposes of presentation, the patch from the source image was 3D rendered at the required slant using OpenGL, and then presented through a 3D-rendered circular aperture with radius $11.25 \mathrm{~cm}$ on the screen (397 pixels), surrounded by a dark-gray area that filled the screen. Under our image-generation approach, a key property of the image is that it must always contain higher frequency content (smaller edge fragments) than can be visually perceived at the maximal zoom level. During the OpenGL rendering process, each of the computer monitor's physical pixels within the viewing aperture must correspond to one or more generated pixels in the underlying image patch, with a many-to-one correspondence being preferable to avoid potentially substantial down-sampling artifacts. This was accomplished by using patches that were 4,096 pixels on a side in the source image. When projected by Open GL rendering to the screen, they were locally sub-sampled by a factor ranging from .35 to .43 , depending on slant. The radius of the circular aperture was sufficiently small that the edges of the square patch behind it were not exposed even at the most extreme slant, $30^{\circ}$. The subject was placed sufficiently far from the screen $(50 \mathrm{~cm})$ so that the individual pixels on the computer monitor corresponded to $<2$ minutes of arc per pixel and could not be resolved under the experimental conditions.

There were two viewing conditions. In the Stereo condition, the participant viewed the stimulus (slanted or flat) with stereoscopic cues controlled by an Open-GL program using parallel axis asymmetric frustum perspective projection. The left and right "virtual camera" images for each eye were simulated using an eye separation with an inter-axial separation of $60 \mathrm{~mm}$, and the two eyes were stimulated in alternating frames at $120 \mathrm{~Hz}(60 \mathrm{~Hz}$ per eye). Figure 7 shows a stereo pair.

In the condition contrasted with stereo, the participant viewed an invariant frontal projection of the corresponding stimulus with both eyes, in essence showing both eyes the exact same perspective and pixels that would have been seen by a single eye in the stereo condition. We will label this binocular condition Mono, with the understanding that the same monocular cues are seen by both eyes. Although this condition constituted a cue-conflict situation (as described in the introduction), incorporation of the "tiles" images provided direct comparisons between a well- 
studied grid stimulus (tiles) vs. our present stimulus for both pictorial and pictorial+stereo viewing conditions.

\subsection{Design}

Stimuli within each viewing condition were presented at one of 5 slant directions: flat, pitch rotated with the top in or bottom-in, and yaw rotated with the right in or left in (where "in" refers to into the screen, further from the viewer). The 4 nonflat slants could take on magnitudes of $15^{\circ}$ or $30^{\circ}$.

The trials were divided into 4 blocks that alternated between Mono and Stereo viewing; the two possible viewing orders were counterbalanced across participants. Within each block there were 70 experimental trials: These included 60 trials with the generated set, 12 at each slant direction. Within each slant direction other than flat, the two magnitudes $\left(15^{\circ}\right.$ or $\left.30^{\circ}\right)$ occurred equally often, and the values of region and wrapping parameter were represented equally within each condition. The remaining 10 trials in a block presented tiles, two at each slant direction (one for each magnitude) and two flat. The trials were randomly ordered within each block. Before the first block, there was a 20-trial practice block with 5 trials in each viewing condition, in the same order as would follow in the experiment proper, and each successive block was preceded by 5 practice trials, 1 at each slant.

A pool of 280 unique stimuli was created for the experiment, 240 drawn from the generated set and 40 from a source image of tiles, representing the constraints of the stimulus variables. Once the sequence of trials was determined by the control program for a given subject, a stimulus of the appropriate type was drawn from the pool and presented with the designated viewing condition and orientation. Thus each subject saw a unique order of stimuli, and the same source stimulus occurred randomly across experimental conditions.

\subsection{Procedure}

After informed consent, the participant was administered the stereo acuity test. He or she was then seated at a Dell computer with a $47.5 \mathrm{~cm}$ x $29.8 \mathrm{~cm}$ Samsung Syncmaster 2233RZ LCD screen, so that the head was at an optimal viewing distance for the stereo display $(50 \mathrm{~cm})$, as aligned with a mark on the adjacent 
wall. The participant was told that he or she could move the head laterally but not forward or backward, and the alignment was checked periodically by the experimenter. Each trial began with a signal cross that was viewed for $500 \mathrm{~ms}$, followed by exposure of the stimulus. When ready to respond, the participant pressed a key on a number pad to indicate its slant direction, at which point the selected key input and its corresponding slant were displayed on the screen for confirmation or correction if mistyped (responses were typically made without looking at the keypad, and occasionally the participant wished to correct a known erroneous response). The response keys were arranged in a cross for intuitive mapping to the slant options (e.g., the left arm of the cross corresponded to left edge inward, etc.) Participants had up to $7 \mathrm{sec}$ to make the response.

\subsection{Results}

Initial analyses were performed to test whether the region variable (distance of patch from slant axis in source image) had a significant effect on accuracy (proportion correct on orientation out of 5 choices). Separate analyses of variance for the Mono and Stereo data showed no effect of region, ps > .10. Therefore data were pooled over region for all subsequent analyses.

Table 1 shows the proportion correct for each slant magnitude by viewing condition and stimulus category. As expected, there was a strong tendency to report generated stimuli viewed in the Mono condition as flat (overall, $46 \%$ of responses to generated stimuli, relative to $20 \%$ chance with 5 response alternatives), resulting in the higher accuracy for the $0^{\circ}$ slant in the Mono condition. Considering the $54 \%$ of total responses that were other than "flat," $16 \%$ indicated the stimulus was slanted right edge in, 13\% left in, $8 \%$ top in, and $17 \%$ bottom in. This indicates no particular bias to report pitch (top or bottom) vs. yaw (left or right) slants.

Table 1. Proportion correct identification of slant direction by viewing condition (Mono, Stereo) and stimulus (tiles, generated wrap 1, generated wrap 2) in Experiment 1.

\begin{tabular}{|c|c|c|c|c|c|c|}
\hline & & MONO & & & TEREO & \\
\hline \multirow{2}{*}{ Slant } & \multirow{2}{*}{ Tiles } & \multicolumn{2}{|c|}{ Generated } & \multirow{2}{*}{ Tiles } & \multicolumn{2}{|c|}{ Generated } \\
\hline & & Wrap 1 & Wrap 2 & & Wrap 1 & Wrap 2 \\
\hline
\end{tabular}




\begin{tabular}{|c|c|c|c|c|c|c|}
\hline $0^{\circ}$ & 0.65 & 0.46 & 0.48 & 0.73 & 0.78 & 0.76 \\
\hline $15^{\circ}$ & 0.73 & 0.1 & 0.15 & 0.78 & 0.73 & 0.74 \\
\hline $30^{\circ}$ & 0.94 & 0.13 & 0.22 & 0.95 & 0.90 & 0.90 \\
\hline
\end{tabular}

Initial ANOVAs were conducted with factors of stimulus category (tiles, generated wrap 1 and wrap 2), experimental block (2 levels) and order of conditions (Mono first vs. Stereo first). These analyses were done separately for Mono and Stereo viewing conditions, because Stereo accuracy was generally high (tiles $84 \%$, generated stimuli $81 \%$ and $80 \%$ for wrap 1 and 2). The ANOVA on Stereo showed no significant effects, indicating that the generated stimuli yielded accuracy under stereo viewing equivalent to the tiles, where perspective cues were also present ${ }^{2}$. The ANOVA on Mono showed only a significant effect of stimulus, $\mathrm{F}(2,20)=330.30, \mathrm{p}<.001$, with the tiles $(80 \%$ correct $)$ superior to both of the generated stimulus categories (18\% and $24 \%$, respectively). Accuracy with tiles did not differ in the Mono and Stereo condition (by t test, $\mathrm{p}=.30$ ).

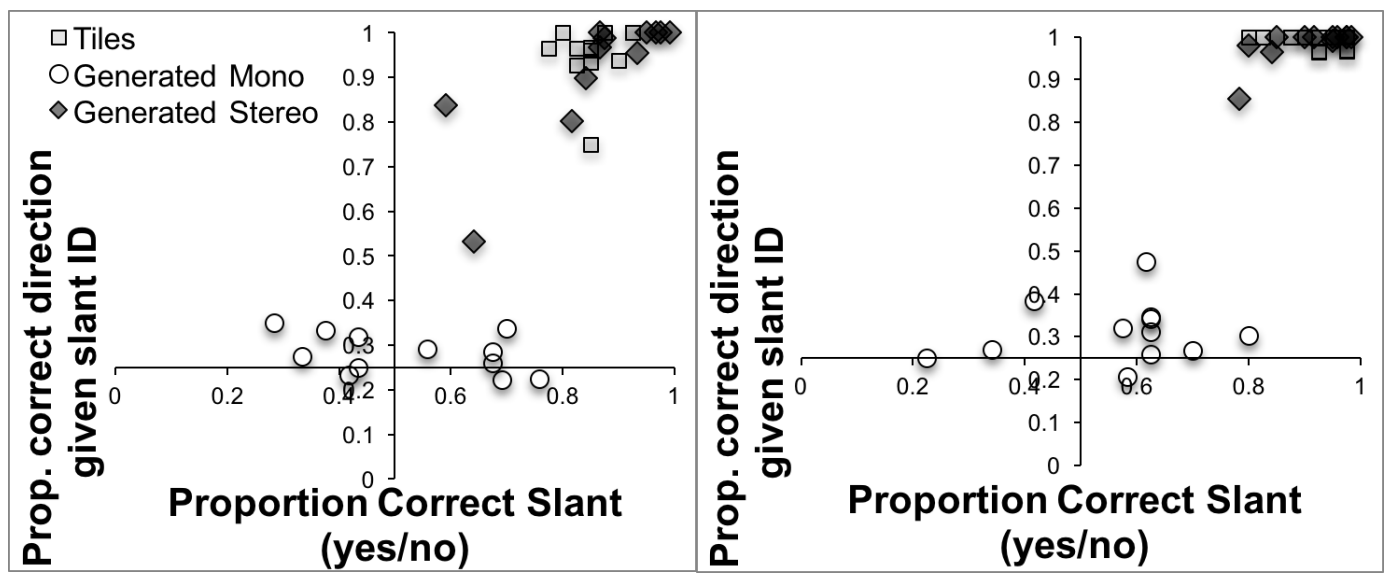

Figure 8. Left: accuracy in Experiment 1 for individual subjects, as assessed by two measures: the discrimination of slanted from non-slanted stimuli ( $\mathrm{x}$ axis) and, conditional on accurate identification of slant, the identification of stimulus orientation direction (y axis). Both measures pool data from $15^{\circ}$ and $30^{\circ}$ slants. The axes are adjusted to cross at chance for both measures. Right: an equivalent graph for Experiment 2.

${ }^{2}$ Because performance was essentially ceiling-level in these 5-alternative judgments, the data did not manifest a commonly found anisotropy in slant perception with metric responses, i.e., superior performance in matching pitch relative to yaw (e.g., [15]). 
Figure 8 shows the accuracy for each participant in Experiment 1 (left) and Experiment 2 (right, discussed in the next section). Clearly, the generated planar stimuli convey slant under stereo viewing, whereas Mono accuracy clusters near chance.

To assess the effect of slant magnitude, the conditions with slanted stimuli were subjected to 3 X 2 (stimulus: tile vs. generated wrap 1 vs. generated wrap $2 \mathrm{X}$ angle: $15^{\circ}$ vs. $30^{\circ}$ ) ANOVAs, separately for Mono and Stereo viewing conditions. In the Mono condition, there were main effects of stimulus, $\mathrm{F}(22)=302.04$, $\mathrm{p}<$ .001 reflecting the obvious superiority of the tiles, which provided perspective cues. There were also effects of angle, $\mathrm{F}(1,11)=56.04, \mathrm{p}<.001$, and an interaction, $F(2,22)=6.01, p=.008$. The latter effects arise from an advantage for the $30^{\circ}$ angle for tiles (by t test, $\mathrm{p}<.001$ ), but not generated stimuli. In the Stereo condition, the sole effect was of angle, $\mathrm{F}(1,11)=28.90, \mathrm{p}<.001$. This indicates that under stereo viewing, the generated stimuli and tiles were equally well identified as to orientation and showed an equivalent advantage for the $30^{\circ}$ angle over the $15^{\circ}$.

The remaining analyses focused on the generated stimuli in the Mono conditions, where unlike the Stereo conditions or tiles viewed as Mono, substantial errors were made. To assess whether flat and slanted stimuli (in any direction) were discriminated, the d' statistic for flat/slant choices was computed for each subject, and t-tests were used to compare the values to zero. (This measure is independent of differential response frequencies.) Mean d' values at $15^{\circ}$ were .02 and .01 for stimuli generated with algorithms 1 and 2 , respectively, and at $30^{\circ}$ the corresponding values were .02 and .21. None significantly exceeded zero, although the value for wrap 2 at $30^{\circ}$ approached significance, $\mathrm{t}(11)=1.72, \mathrm{p}=.06$ with a 1-tailed test. Thus, as shown by the clustering of data near chance on the $\mathrm{x}$-axis of Figure 8, generated stimuli rendered as slanted vs. flat were essentially indiscriminable under Mono viewing.

The next analysis focused on the data represented by the y-axis of Figure 8, namely, accuracy on identifying the orientation of slant, given that a stimulus was identified as slanted at all. Mean accuracy on slant direction was tested against 
chance $(25 \%)$ by t-test. Only the accuracy for generated wrap 2 at $30^{\circ}$ (mean $38 \%$ ) exceeded chance, $\mathrm{t}(11)=3.45$, one-tailed $\mathrm{p}=.003$. Thus, for the generated stimuli using wrap 2 and subjected to $30^{\circ}$ rotation, once identified as slanted they could be judged with respect to the direction at a level above chance.

Finally, the slant information available from the Mono rendered generated stimuli was further quantified in bits by an information-transmission statistic, which takes into account the response tendencies (Tan et al., 1997). The value was .02 bits for wrap 1 at both orientations and also .02 bits for wrap 2 at $15^{\circ}$, but reached .04 bits for wrap 2 at $30^{\circ}$, again pointing to some orientation information available from this wrapping algorithm under the higher slant.

Although instructions did not indicate responses should be speeded, it is also of interest as to whether the time to respond varied with the stimulus and experimental variables, particularly for the conditions where stereopsis must be induced. An ANOVA with stimulus category (tiles, generated wrap 1 and 2), experimental block (2 levels), and order of conditions (Mono first vs. Stereo first) was conducted on response latency. The Mono condition showed only an effect of stimulus, $\mathrm{F}(2,20)=5.52, \mathrm{p}=.012$ : Responses to tiles (mean latency $=2.5 \mathrm{sec}$ ) were faster than to generated stimuli (mean latency $=3.3 \mathrm{sec}$ for each wrapping). The response latencies in the Stereo condition showed a stimulus X block interaction, $F(2,20)=5.60, p=.012$, reflecting a drop in response latency for the generated stimuli, but not the tiles (tiles $1.9 \mathrm{sec}$ in both blocks, generated stimuli decreased from $2.0 \mathrm{sec}$ to $1.6 \mathrm{sec}$ from Block 1 to Block 2). Importantly, the time to elicit a response under stereo viewing was no greater for generated stimuli than for tiles.

In short, the analyses indicate first that under stereo viewing, the generation algorithm produced planar stimuli that were quickly and accurately identified as to slant direction, equally so to stimuli that offered perspective cues. Second, multiple measures indicate that when viewed without stereo cues, stimuli generated with wrap 1 provided essentially no information about slant, even at $30^{\circ}$. However, stimuli generated with wrap 2 and presented at $30^{\circ}$ did provide non-stereo slant cues with measurable impact, albeit small. Specifically, when 
using wrap 2 there was (a) marginally significant slant/non-slant discrimination, and (b) significantly above-chance identification of direction of orientation for stimuli identified as slanted.

\section{Experiment 2}

The randomized assignment in Experiment 1 of source stimuli to viewing condition and slant means that the obtained level of slant perception does not reflect the contribution of a single stimulus. However, it remains possible that under non-stereo viewing, some individual stimuli generated by our process might have reliable slant cues or that the process might bias an illusory slant. To assess these possibilities, Experiment 2 obtained multiple responses to the same stimulus within a condition.

\subsection{Method}

The participants were 12 young adult volunteers who gave informed consent. The design of Experiment 1 was repeated, except that rather than assigning a stimulus patch randomly to a viewing condition and slant, a given patch was assessed at the same slant direction and magnitude across all subjects within a viewing condition. This resulted in six participants providing responses to the same stimulus at a particular slant direction and magnitude, with 48 generated stimuli being assessed in the flat orientation and 96 in the slanted.

\subsection{Results}

Individual subjects' proportions correct in slant presence and direction for all conditions for this experiment are shown in Figure 8 (right), for purposes of comparison to Experiment 1 . The same pattern can be seen as previously, such that Stereo slant perception is generally excellent, as is perception of tiles without stereo cues, but the generated stimuli produce near-chance performance under Mono viewing conditions.

Because the main purpose of Experiment 2 was to test whether individual generated stimuli might offer reliable or biased non-stereo slant cues, the results of interest are the distribution of responses to a generated stimulus across the five response alternatives in the Mono condition. The analysis assesses whether the observed use of the response options is random or whether, alternatively, 
orientations of some stimuli are judged easily or consistently at some slant. Either of these latter alternatives would produce convergence on a single response.

In fact, only two of the generated stimuli rendered as Mono produced a common response by all subjects viewing them, and that response was incorrect and not biased toward a slant (i.e., "flat") in both cases. To statistically test whether the response choices to generated stimuli in the Mono condition were randomly distributed across the five slant alternatives, the number of unique slant responses used by the six subjects viewing a single stimulus was determined (regardless of which particular responses were chosen). For example, if three subjects chose top-in and three chose flat, that would constitute two unique responses out of the five possible for that stimulus. The empirical distribution of the number of unique responses was computed for generated stimuli rendered as Mono, separately for sets with objective slants of $0^{\circ}$ (flat), $15^{\circ}$ and $30^{\circ}$. To assess whether these observed distributions of unique responses reflect random responding, Monte Carlo simulations were used to generate a random distribution for comparison. In each simulation, six random choices were made out of five alternatives for a given stimulus (representing the six participants and the five slant alternatives), where the probabilities of the slant responses were set to the marginal response tendencies that had been exhibited empirically. This was repeated for the total number of stimuli of the given slant magnitude, thus generating a distribution of number of unique slant responses based on random responding. One hundred such simulations were run for each slant magnitude, and the $95 \%$ confidence interval around the mean value of each number of unique responses was calculated. All 15 comparisons between observed and simulated values ( 5 numbers of unique slant responses X 3 slant magnitudes) were consistent with random responding (i.e., the observed proportion of that number of unique choices fell within the $95 \%$ confidence interval around the simulation mean). The same results held when the two generated subsets defined by wrap algorithm were assessed separately. Thus the tests clearly indicate that for the population tested, Experiment 1's aggregate data indicating ineffectiveness of non-stereo cues are not masking generated stimuli that are highly informative or biased on this basis. 


\section{Discussion}

The present algorithm largely met our goal of generating planar images that eliminate gradient cues when slanted (either in a 3D rendering or in a physical instantiation) but allow for stereoscopic depth processing. Under one parameterization, the algorithm yielded no evidence of Mono slant detection, let alone identification. The alternative parameterization appeared to allow a small amount of information to "leak" through under Mono viewing at the maximum slant; however, the ability to discriminate slant from flat still did not statistically exceed chance. Experiment 2 demonstrated that these results were not the result of aggregating data across stimuli affording good and bad levels of slant perception.

The data showed a small but reliably detected level of pictorial gradient cues for wrap 2, for which the dynamic range of the images was the lesser. Further work is needed to establish why the lesser dynamic range (a reduction of information) somewhat counter-intuitively led to better (i.e., barely measurable) perception of slant. One possibility is that the higher contrast of wrap 1 allowed higherfrequency content to be perceived, at least at extreme levels, producing a masking effect [16]. It also remains to be determined whether the subtle gradient cues that emerged with wrap 2 are from edge statistics (which in theory, at least for the underlying triangular edges, should have been fully controlled) or some other monocular source (e.g., distributions of locally average intensities in the mid to low frequencies). These issues notwithstanding, we have demonstrated one algorithm that was completely successful by the present criteria, and another that offered very little monocular information regarding slant.

\section{Conclusion}

The chief contribution of this research was to devise an algorithm for generating static planar images that when converted to viewed displays, offer essentially no pictorial gradient cues to slant while affording stereo vision. Unlike previous such efforts (e.g., [7]), a further requirement was that the images should be practically realizable as physical objects by printing. The approach taken here was to populate an image with bounded triangular shapes at essentially continuous distribution of scale, with sufficient density to maintain essentially constant highfrequency edge statistics over a range of magnification and other forms of 
zoom/cropping, and to degrade low-frequency content so as to suppress any perceivable texture gradients. The results demonstrated that this goal could be met for slants up to $30^{\circ}$ within the experimental constraints: Stereoscopic observation of stimuli generated with the algorithm allowed participants to perceive slant correctly, but monocular observation left them essentially uninformed, even about its direction.

\section{Acknowledgements}

Bing $\mathrm{Wu}$ is thanked for valuable comments on the project and manuscript.

Funding: This work was supported by the National Institutes of Health [grant number R01EY021641] and by a National Science Foundation Graduate Research Fellowship [under grant number DGE-1252522]. Neither funding source was directly involved in any aspect of this work.

\section{Vitae}

John Galeotti received BS and MS degrees in Computer Engineering from North Carolina State University (2001, 2002), and MS and PhD degrees in Robotics from Carnegie Mellon University $(2005,2007)$. He is currently a systems scientist at Carnegie Mellon University's Robotics Institute, as well as an adjunct assistant professor with advising privileges at both CMU's Biomedical Engineering department and at the University of Pittsburgh's Bioengineering department. He directs the Biomedical Image Guidance Laboratory at CMU, and he teaches an internationally recognized graduate course on biomedical image analysis algorithms. He is a member of the IEEE.

Kori Macdonald received a bachelor's degree from the University of Pittsburgh in bioengineering, with a research interest in understanding depth perception. She received both a Master's in Mechanical Engineering with a focus in robotics, and a Master's in Business Administration, from Rice University. She is currently working with GE Global Research in Niskayuna, NY in the Edison Engineering Development Program. 
Jihang Wang received the master's degree in Biomedical Engineering from Carnegie Mellon University in 2012. He is currently a Ph.D. candidate in Bioengineering at the University of Pittsburgh with particular interests in biomedical computer vision, medical image analysis, computer graphics and visualization.

Samantha Horvath received the M.S. degree in robotics from Carnegie Mellon University, where she is currently pursuing the Doctoral degree with the Robotics Institute. Her research interests include medical device design and medical image analysis, with a focus on computer vision and optical engineering. She has done research on image-guided ultrasound and augmented reality systems for surgical microscopes.

Ada Zhang received her B.S. in Bioengineering from the University of Washington and has been a doctoral student in the Robotics Institute since August 2011. She is a recipient of the NSF Graduate Research Fellowship. Her broad areas of interest lie at the intersection of healthcare and engineering.

Roberta Klatzky received the B.S. in mathematics from the University of Michigan and Ph.D. in cognitive psychology from Stanford University. She is currently the Charles J. Queenan, Jr. professor of psychology and humancomputer interaction at Carnegie Mellon University. Her research interests include human perception and cognition, with emphasis on touch, spatial cognition, and perceptually guided action. Her work has been applied to navigation aids for the blind, haptic interfaces, exploratory robotics, image-guided surgery, and virtual environments. She is a Fellow of the American Association for the Advancement of Science, the Assocation for Psychological Science, and other professional organizations.

\section{References}

[1] J. J. Gibson, The Perception of the Visual World, Haughton Mifflin, Boston, 1950.

[2] W. P. Purdy, The hypothesis of psychophysical correspondence in space perception, Dissertation Abstracts International, 42, 1454, 1958 (University Micro- films No. 58-5594). 
[3] J. T. Todd, L. Thaler, T. M. H. Dijkstra, J. J. Koenderink, A. M. L. Kappers, The effects of viewing angle, camera angle and sign of surface curvature on the perception of 3D shape from texture, Journal of Vision, 12 (2007) 9, 1-16.

[4] J. T. Todd, L. Thaler, The perception of 3D shape from texture based on directional width gradients, Journal of Vision, 5 (2010) 17, 1-13.

[5] A. P. Witkin, Scale-space filtering, In Proceedings of the Eighth international joint conference on Artificial intelligence - Volume 2 (IJCAI 1983), Morgan Kaufmann Publishers Inc., San Francisco, CA, USA, 1019-1022, http://ijcai.org/Past\%20Proceedings/IJCAI-83-VOL2/PDF/091.pdf.

[6] J. J. Koenderink, The structure of images, Biological Cybernetics, Volume 50 Issue 5 (August 1984), 363-370, http://dx.doi.org/10.1007/BF00336961

[7] X. Zabulis, B. T. Backus, Starry night: A texture devoid of depth cues, Journal of the Optical Society of America, 11 (2004), 2049-2060.

[8] D. Marr, T. Poggio, A computational theory of human stereo vision, Proceedings of the Royal Society (London), Series B, 204 (1979), 301-328.

[9] B. Gillam, Judgments of slant on the basis of foreshortening, Scandinavian Journal of Psychology, 11 (1970), 31-34.

[10]B. B. Mandelbrot, Fractal aspects of the iteration of $\mathrm{z} \rightarrow \lambda \mathrm{z}(1-\mathrm{z})$ for complex $\lambda$ and $\mathrm{z}$, Annals of the New York Academy of Sciences, 357 (1980), 249-259.

[11] W. Sierpiński, Sur une courbe dont tout point est un point de ramification, Computational Rendus Academy of Science Paris, 16, (1915), 302-305.

[12] V. A. Billock, Neural acclimation to $1 / f$ spatial frequency spectra in natural images transduced by the human visual system, Physica D, 137 (2000), 379-391.

[13] D.C. Knill, D. Field, D. Kersten, Human discrimination of fractal images, Journal of the Optical Society of America, 7 (1990), 1113-1123.

[14] M. Haidekker, Advanced Biomedical Image Analysis, John Wiley \& Sons, New York, 2011.

[15] B. Gillam, C. Ryan, Perspective, orientation disparity, and anisotropy in stereoscopic slant perception. Perception, 21 (1992), 427-439.

[16] L. D. Harmon, B. Julesz, Masking in visual recognition: Effects of two-dimensional filtered noise, Science, 180 (1973), 1194-1197. 
Novel images lack monocular (pictorial) depth and slant cues while containing rich information for stereopsis.

Control images do not prevent monocular slant perception.
Top slanted away $30^{\circ}$

Flat
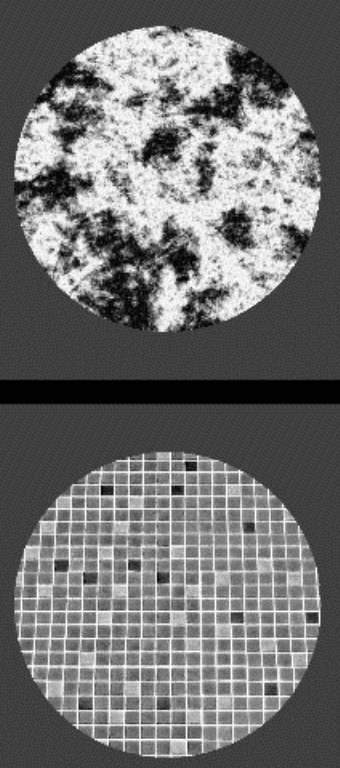

Bottom slanted away $30^{\circ}$
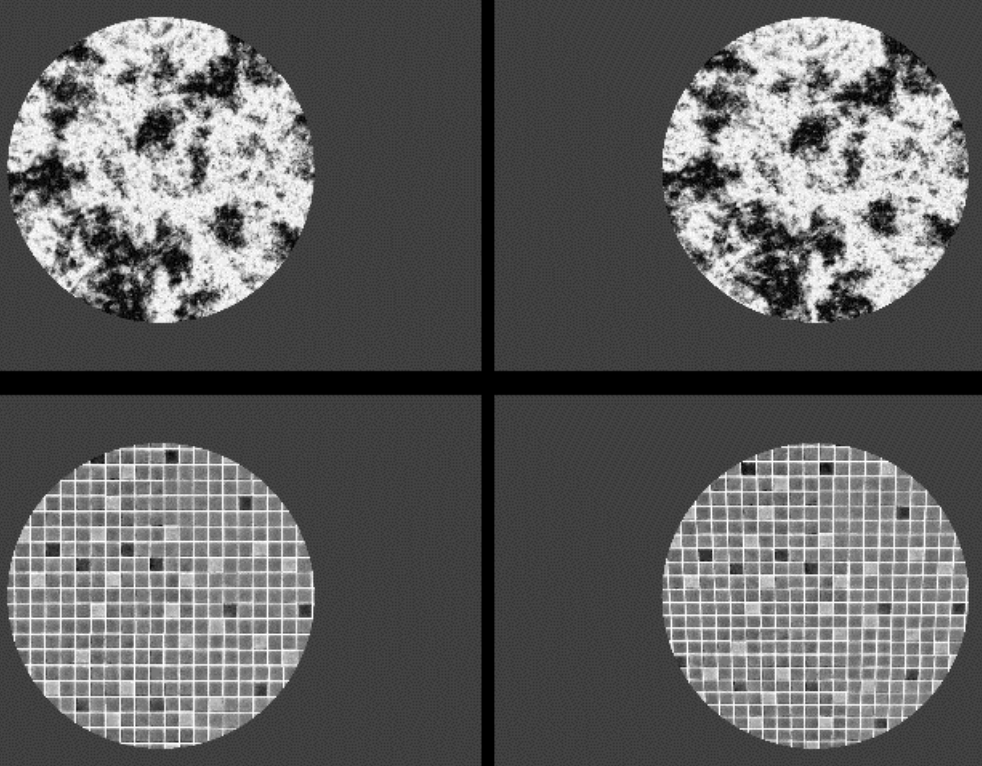